\title{
ANT COLONY OPTIMIZATION BASED MODIFIED TERMITE ALGORITHM (MTA) WITH EFFICIENT STAGNATION AVOIDANCE STRATEGY FOR MANETS
}

\author{
Sharvani G S, Dr. A G Ananth and Dr T M Rangaswamy \\ R V College Of Engineering \\ Mysore Road, Bangalore \\ sharvanigs@rvce.edu.in, antisro@yahoo.com, rangaswamytm@rvce.edu.in
}

\begin{abstract}
Designing an effective load balancing algorithm is difficult due to Dynamic topology of MANET. To address the problem, a load balancing routing algorithm namely Modified Termite Algorithm (MTA) has been developed based on ant's food foraging behavior. Stability of the link is determined based on node stability factor ' $\Delta$ '. The stability factor " $\Delta$ "of the node is the ratio defined between the "hello sent" and "hello replied" by a node to its neighbors. This also indicates the link stability in relation to other paths towards the destination. A higher ratio of " $\Delta$ " indicates that the neighbor node is more stable. Using this concept pheromone evaporation for the stable node is fine tuned such that if the ratio " $\Delta$ " is more, the evaporation is slow and if " $\Delta$ " is less the evaporation is faster. This leads to decreasing of the pheromone content in an optimal path which may result in congestion. These paths can be avoided using efficient evaporation technique. The MTA developed by adopting efficient pheromone evaporation technique will address the load balancing problems and expected to enhance the performance of the network in terms of throughput, and reduces End-to-end delay and Routing overheads.
\end{abstract}

\section{KEYWORDS}

Ant colony optimization, MANET, Node stability, Termite Algorithm, stagnation

\section{INTRODUCTION}

Data transfer on the move is highly essential and is the basic need in MANETs. Mobility of nodes in MANETs affects the performance of routing protocols. Using greedy mechanism, the network tends to choose a single path every time which leads to congestion. Designing an effective load balancing algorithm is difficult due to dynamic topology of MANET. To address this problem, a load balancing routing algorithm Modified Termite Algorithm (MTA) has been developed based on ant's food foraging behavior.

The MTA developed by adopting efficient pheromone evaporation technique will address load balancing problems and will enhance the performance of the network in terms of throughput, and reduces End-to-end delay and routing overheads. 
International journal on applications of graph theory in wireless ad hoc networks and sensor networks (GRAPH-HOC) Vol.4, No.2/3, September 2012

\subsection{Mobile Ad-hoc Networks (MANETs)}

Wireless communications can be categorized into Infrastructure dependent and infrastructure independent networks. The Cellular networks are infrastructure dependent where as Ad hoc Wireless Networks (AWN) are capable of operating without any fixed infrastructure support. MANET is one such category of AWN, which has a set of mobile agents, communicating with each other with-in radio frequency range. Since radio frequency range is limited, the communication traffic has to be relayed over several intermediate nodes. Therefore, MANET is also known as Multi Hop ad hoc networks. The functionality of the nodes is not only to fulfill as a host, but also as a router to forward packets to other nodes. Routing in MANET is a challenging task due to characteristics like node mobility - which leads to dynamic Topology, Error prone shared channel, channel contention and limited device availability. Hence, an efficient routing protocol has to be developed which is capable of reducing control overhead and utilizes the bandwidth efficiently [1] [2]. Ant Colony Optimization (ACO) is Swarm Intelligence (SI) based routing algorithm proposed for high quality routing in MANET.

\subsection{Ant Colony Optimization (ACO)}

ACO was inspired by an algorithm called "Ant System (AS)" [3]. ACO deals with artificial systems that are inspired from food foraging behavior of real ants, which can find optimal solutions in highly dynamic environment. A major problem with ACO algorithm is "stagnation". This occurs when all ants try to follow the same path to reach the destination (since there is more pheromone). This when applied in MANETs comes to a convergence state (equilibrium) and attracts all the data packets to follow the same path, which leads to congestion.

To mitigate the above problem of ACO algorithms, different methodologies where adopted and are categorized as follows.

- Privileged pheromone Laying

- Pheromone-heuristic control

- Pheromone Control.

The proposed work uses Pheromone control technique in the form of controlled evaporation for efficient routing.

Wireless networks such as MANETS have many advantages compared to wired networks. In MANETs the communication is not limited to a certain region (as in cellular region). MANETs have some peculiar routing challenges. Swarm Intelligence (SI) based routing algorithms provides an interesting analogy to the world of biology. SI based ACO algorithms provide interesting solutions to network routing problems. ACO based routing in MANETs will enhance the reliability and efficient packet delivery. They help in reducing control overhead due to their inherent scalable feature. The similarity between ant and nodes, colony and Wireless network helps to use ACO based routing in MANETs. Termite is one such ACO based algorithm which performs better than the AdHoc On demand Distance vector (AODV).

\section{Stagnation avoidance algorithms}

Elitist Ant System (EAS) algorithm is a variation of ACO [4]. Here pheromone deposits are only for the best path found. The major problem with this algorithm is early stagnation. Max-Min Ant systems (MMAS) [5] overcome the early stagnation problem by adding an extra constraint which says that the pheromone is bounded between maximum and minimum pheromone concentration. 
International journal on applications of graph theory in wireless ad hoc networks and sensor networks

(GRAPH-HOC) Vol.4, No.2/3, September 2012

Stagnation avoidance algorithm was developed while ACO found its application in Scheduling of flexible manufacturing systems [6]. The methodology developed deals with quick convergence and stagnation avoidance in which parameters of ACO such as evaporation co-efficient which control the trail and its visibility are fine tuned. MMAS was further improved by adopting Minimum Pheromone Threshold Strategy (MPTS)[7]. Here the bound between maximum and minimum threshold is fine tuned based on performance of the network. Improved Lower Limits for Pheromone Trails in ACO adopts improved estimates of the lower pheromone value. This helps algorithms like MMAS (sets implicit pheromone trail limit) to avoid stagnation [8]. Another work on stagnation avoidance for scheduling of real-time tasks [9] uses a non-preemptive scheduling approach based on distance function as an extra parameter in the transition rule with the pheromone information.

A framework for stagnation avoidance was developed while adapting ACO in TSP [10]. This frame work evaluates each solution against the last "N" solutions globally constructed by ants. As soon as " $\mathrm{N}$ " solutions are available, their moving average " $\mathrm{A}$ " is computed. New value of $\mathrm{A}$ is compared with old value. If New A is lower than old, the trail level of the last solution's moves is increased, otherwise it is decreased.

An ACO algorithm with improved pheromone correction strategy for the Minimum Weight Cover Problem (MWCP) [11] [12] explains that new Hybridization techniques. Corrections to the pheromone trails are performed based on suspicion whether it is good or bad. It decrements the pheromone trails very fast if it finds more suspicious elements. In Hybrid architecture, the age of the ant decides whether the trail should be increased or not [13]. Another solution for stagnation was found by using information entropy [14] which helped the algorithm to fine tune its pheromone trails for local search.

\section{Termite Hill Building process}

Termite's hill building process involves, collecting the pebbles spread over an area and place it in one place. The cooperation between Termites will lead to constructing of a huge hill (as big as 7 Feet). This is achieved by Termites moving on locally observed pheromone trails (laid by other Termites).If no trails are found, it moves randomly in any direction.

As shown in Fig 1 each Termite may carry only one pebble at a time and move observing the trail laid by other Termites. If a pebble is found on the way it just puts down pebble carried by it and both pebbles are infused with pheromone which then evaporates.

Termite's inclination towards pheromone trail (positive feedback) leads to large piles of pebbles forming a hill. At the same time evaporation (negative feedback) declines the movement of Termite towards the direction in which hill building is not appropriate or avoids small piles. It is essential that many individual work together, otherwise before the pebbles added to the pile the evaporation of trail leads random walk by Termites without forming any significant piles.

Analogous to the above example, each node in the Mobile wireless network is a Termite Hill. As packets travel from source to the destination, it leaves pheromone for its source and at the same time follows the pheromone for its destination through the MANET. The 
International journal on applications of graph theory in wireless ad hoc networks and sensor networks

(GRAPH-HOC) Vol.4, No.2/3, September 2012

pheromone is laid between nodes' links which are communicating with each other. As in Termite example, packets are inclined towards strong pheromone trails. Laying source pheromone trail on the path increases the likely hood of packets travelling on the same path (reverse path).

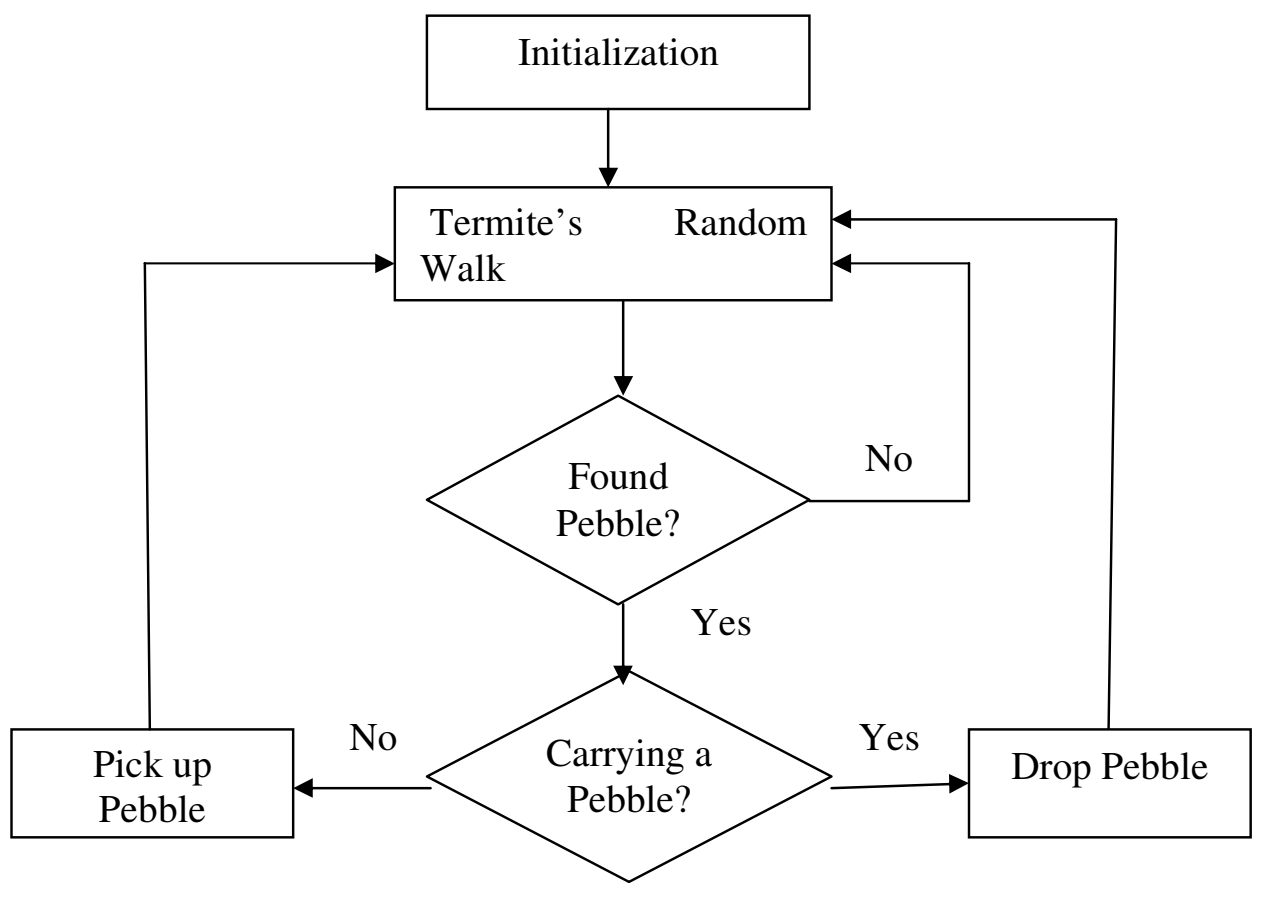

Fig 1 Flowchart of Termite Hill building process

\section{Modified Termite Algorithm (MTA)}

Termite algorithm is a routing protocol for MANET. The packets in the network follow the principles of SI and results in emergent routing behavior. Packets are not forwarded to the same neighbor from whom it received (to avoid cycles or loops). It is similar to ARA in terms of routing data but varies with respect to route discovery and failure handling. It is inspired by hill building procedure

Termites are biased towards the "pheromone" laid by other termites towards the destination hill. This is very similar to packets in MANETs biased towards the optimal path to reach the destination. Information about the network will be carried by data packets which help in making probabilistic routing decision. This decision helps to find maximum utility path in an emergent manner.

As shown in the context diagram Figure 2 the network gives the source and destination node to the Termite Routing algorithm for which the data is to be routed. 


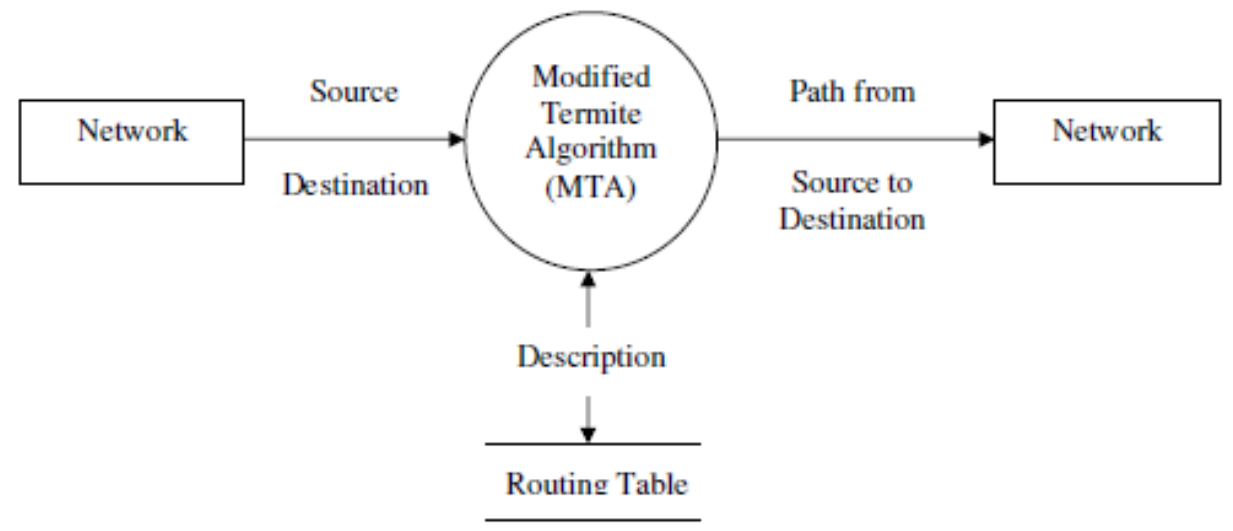

Figure: 2 Routing in MANETs using Termite Algorithm

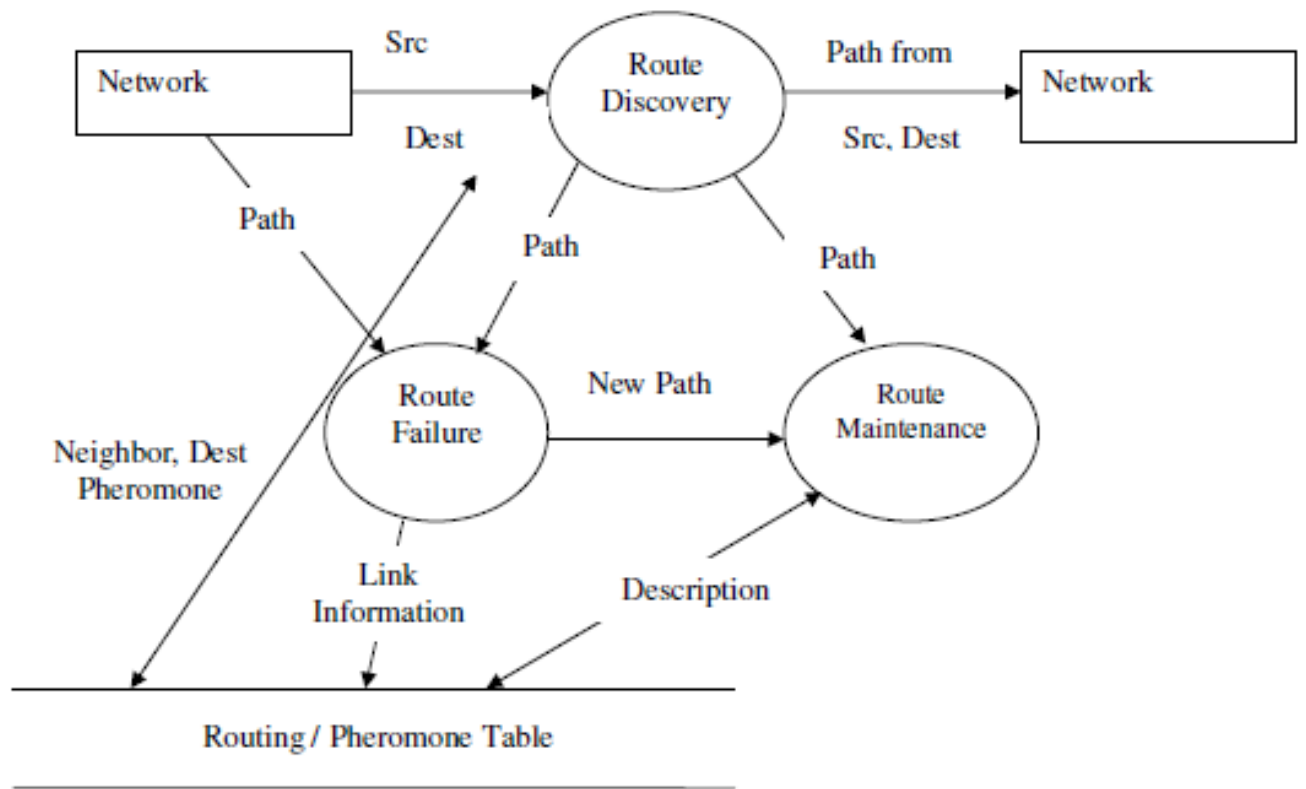

Figure: 3 Phases of MANETs using Termite Algorithm

The algorithm transmits destination, neighbor and pheromone value to and from the routing table i.e. the routing table is updated. The output given is the route from the source to destination through which the data is routed. The context diagram of Figure. 2 gets segregated into several processes as shown in Figure 3. These processes work together to achieve the desired result.

The first process here is the Route discovery which takes the source and destination node from the network. It searches for a path from source to destination. Once the path is 
International journal on applications of graph theory in wireless ad hoc networks and sensor networks (GRAPH-HOC) Vol.4, No.2/3, September 2012

discovered, the best path is maintained by sending data on that path by the Route maintenance module. The third module is for Route Failure to detect for any link failure and to find the new path.

This approach reduces control overhead and maximizes routing performance focusing on the routing metric estimator "pheromone." The pheromone is adjusted in such a way that the best possible utility link is chosen for delivering the packets to the destination.

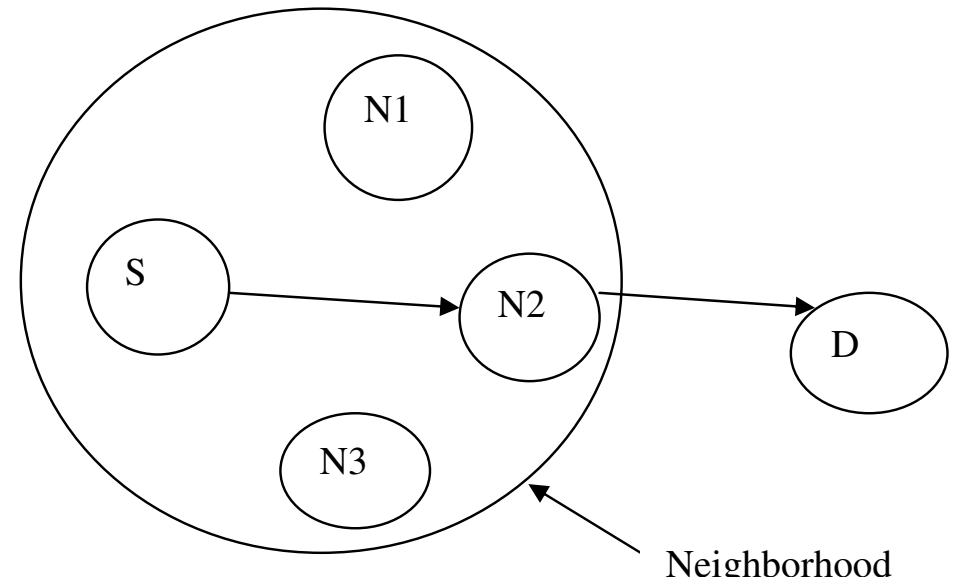

Fig 4'S' node neighborhood

\subsection{Routing in MANETs with MTA}

Before routing the network has to stabilize itself by sending hello packets (or through sniffing) to its neighbors and build the routing or pheromone table. This helps them to know their neighbors. Neighbor nodes must be carefully handled as they can forward/ originate packets. However, they always give preference for packets to be forwarded. Thus, the neighbor row must be declared decayed before the neighbor node can be removed from the pheromone table. If a neighbor is determined to be lost by means of communications failure, the neighbor row is simply removed from the pheromone table. Similarly a decayed column indicates that no traffic has been seen which was sourced by that node.

\subsubsection{Routing table in MTA}

Termite's pheromone table is analogous to routing table. Each node in the network maintains a pheromone table which keeps track of percentage of pheromone in each neighbor link. The table consists of row representing the neighbors and columns correspond to destinations (except for node itself). Table size is dynamic and varies based on the destinations heard recently. An entry of node ' $\mathrm{n}$ ' in the table is referenced by $\boldsymbol{P}_{i, d}^{n}$ where $i \in N^{n}$ is the neighbor and 'd' denotes the destination where $d \in D^{n}$, in other words, $P_{i, d}^{n}$ is the percentage of pheromone from a node 'd' on the link with neighbor ' ' $\mathrm{i}$ at node 'n'. 
International journal on applications of graph theory in wireless ad hoc networks and sensor networks

\subsubsection{Pheromone update/Decay}

(GRAPH-HOC) Vol.4, No.2/3, September 2012

Pheromone is updated only by data packets as compared to other ACO based algorithm in which all forward ants, backward ants and data packets update pheromone concentration. When a packets arrives at a node ' $\mathrm{n}$ ' from source $\mathrm{s}$ and a previous hop 'p', the new pheromone concentration entry for $P_{p, s}^{n}$ for the source of the packet is increased by constant pheromone value ' $\gamma$ ' equation as shown in Eqn (1) the.

$$
P_{p, s}^{n} \leftarrow P_{p, s}^{n}+\gamma \ldots \ldots \ldots \ldots \ldots \ldots . \text { Eqn (1) }
$$

Pheromone decay is an important factor for efficient packet delivery, if not handled properly, it leads to stale entries in the network which lead to packet drops or it might lead to improper load in the network due to data packets travelling on the optimal path and increasing the pheromone concentration. This tells other packets to travel blindly not knowing whether the link is overloaded or not.

Pheromone values in the routing table will be decayed periodically as shown in Eqn (2) by multiplying it with decay factor, $\mathrm{e}^{-\tau}$ where $\tau \geq 0$ and is a global parameter. There should be a balancing act between the pheromone decay process i.e. neither too quick nor slow. The nominal pheromone decay interval is one second which is called as decay period.

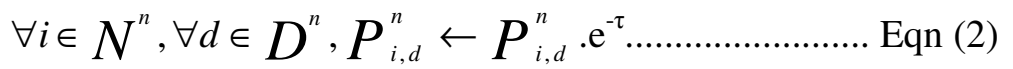

If all the pheromone for a particular node (as it has not received any packets for a long time) decays, the row for that is removed from the routing table. However, column entry cannot be deleted directly if it is also neighbor node. Pheromone bounds like max, min and initial pheromone values (prevents extreme difference in pheromone value) in the routing table plays a major role in stagnation problems. Table updates new column and row for the neighbor as it can also be a destination. When a packet is received from unknown source then the new entry for that node is created on the routing table. If the nodes are just destinations then only the column are created.

For example as shown in Fig 4, Source ' $\mathrm{S}$ ' considers N1, N2 and N3 as neighboring nodes since they are within the transmission range. Once the neighboring nodes are known then the routing table is updated as shown in Table 1(route discovery also helps in updating the routing table). When node ' $\mathrm{S}$ wants to communicate with destination ' $\mathrm{D}$ ' it checks for the highest trail for destination ' $\mathrm{D}$ ' and then forwards the packet.

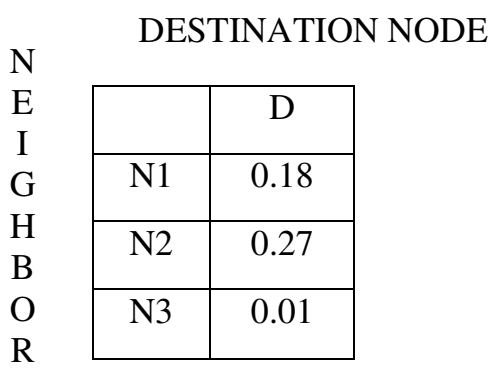

TABLE 1 ROUTING TABLE AT NODE 'S' 
International journal on applications of graph theory in wireless ad hoc networks and sensor networks

(GRAPH-HOC) Vol.4, No.2/3, September 2012

\subsection{Efficient Stagnation avoidance Technique}

Out of the many decay functions analyzed, the exponential decay techniques in ACO are more applicable for dynamic networks like MANETs. The limitation of this technique is pheromone evaporates (uniformly) very quickly after some time leaving no traces for the data transmission. Hence, fresh route discovery is established to know the destination address. This causes more control overhead and makes bandwidth inefficient. To deal with this problem, controlled exponential evaporation is adopted which fine tunes evaporation based on the stability factor ' $\Delta$ ' of the node .Each node in the network also maintains the Node stability Table for all its neighboring nodes as shown in TABEL2. This table helps in finding more stable nodes.

\begin{tabular}{l|l|c|}
\multicolumn{2}{c}{} & \multicolumn{2}{c}{ STABILITY FACTOR } \\
\cline { 2 - 3 } N & & Ratio= Hello sent / Hello replied \\
E & & $(\%)$ \\
I & & 55 \\
G & & 78 \\
\cline { 2 - 3 } H & N1 & \\
B & N2 & \\
O & & 24 \\
\cline { 2 - 3 } R & N3 &
\end{tabular}

TABLE 2 NODE STABILITY FACTOR OF NEIGHBOR NODES AT NODE 'S'

Evaporation is fine tuned based on the stability factor of the node in the vicinity of the node that forwards the packet. During packet forwarding time, each node calculates the 'hello' sent and 'hello' received by its neighboring nodes and calculates the stability ratio shown in Eqn (3)

$$
\text { Ratio }=(\text { Hello Replied } / \text { Hello Sent }) * 100 \ldots \ldots \ldots \ldots \ldots \ldots \ldots \ldots \text {............. (3) }
$$

With the help of ratio calculated, evaporation is controlled for each neighboring node by fine-tuning the decay speed like decaying fast for unstable nodes and decaying little slower for stable nodes .

Exponential Decay may result in faster lost of pheromone trail traces. Pheromone trail is controlled by adding an extra parameter ' $\Delta$ ' to the exponential decay factor.

$$
\text { Where } \Delta= \begin{cases}0.003 & \text { If Ratio is }>75 \% \\ 0.002 & \text { If Ratio is } \leq 75 \% \text { and }<55 \% \\ 0.001 & \text { If Ratio is } \leq 55 \%\end{cases}
$$

This technique helps the MANETs to overcome stagnation problem, it also reduces the control overhead and have efficient packet delivery ratio. ' $\Delta$ ' is tunable based on the application and the node mobility as shown in Eqn 4. This tells with what percentage the link should decay its pheromone (tunable). Table 3 describes pheromone decay factor vs ratio. 
International journal on applications of graph theory in wireless ad hoc networks and sensor networks

(GRAPH-HOC) Vol.4, No.2/3, September 2012

$P_{i, d}^{n} \leftarrow P_{i, d}^{n} \cdot \mathbf{e}^{-(\tau+\Delta)}$

Eqn (4)

\begin{tabular}{|c|c|c|c|}
\hline & If Ratio & If Ratio & If Ratio \\
& $\geq 75 \%$ & $<75 \%$ and $\geq 55 \%$ & $<55 \%$ \\
\hline Decay Factor $\mathrm{e}^{-(\tau+\Delta)}$ & $\left(\mathrm{e}^{-\tau+0.003}\right)$ & $\left(\mathrm{e}^{-\tau+0.002}\right)$ & $\left(\mathrm{e}^{-\tau+0.001}\right)$ \\
\hline
\end{tabular}

TABLE 3 DECAY FACTOR FOR DIFFERENT RATIO

Exponential Decay may result in faster lost of pheromone trail traces. Pheromone trail is controlled by adding an extra parameter ' $\Delta$ ' to the exponential decay factor. Decay should be efficiently handled (at an interval of one second); otherwise a high decay rate will quickly reduce the amount of remaining pheromone, while a low value will degrade the pheromone slowly.

A source ready to forward packet to the destination, takes packet forwarding equation's help to find out the next hop neighbor. This formula (as shown in Eqn (5)) updates pheromone value for that destination' $d$ ' on each outgoing link ' $i$ '. The random selection of next neighbor is performed with the help of packet forwarding equation. Extra care must be taken by not forwarding packet to the previous hop ' $\mathrm{p}$ '.

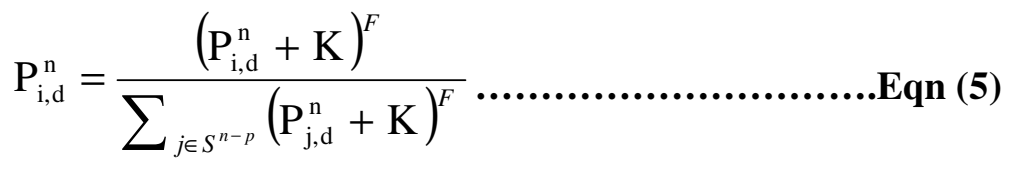

Where F (pheromone sensitivity) and K (pheromone threshold) are tunable based on their sensitivity to amount of pheromone values.

\section{RESULTS AND DISCUSSION:}

The exponential pheromone decay technique based on node stability is adopted which makes the MANET efficient in terms of control overhead, bandwidth, packet delivery etc When applied on Termite algorithm, proved to be better in terms of throughput, end-to-end delay and routing overhead.

Modified Termite Algorithm (MTA) based on ACO has been implemented on MANET. The primary metrics considered for the algorithm are Throughput, End-to-End delay and routing over head against the packet size. A series of tests are conducted to check the feasibility of MTA as an effective mechanism for MANETs based on the community based mobility model. The behavior of success rates with the number of termites (nodes) has been studied. It is observed that only after 30 nodes, the algorithm shows a sharp increase in the success rate (considering $5 \mathrm{~m} / \mathrm{s}$ mobility speed and pause time of 2 secs). It is considered as appropriate to choose the number of termites (Nodes) 30 for determining the throughput, end-to-end delay and control overheads for the MANET. 
International journal on applications of graph theory in wireless ad hoc networks and sensor networks (GRAPH-HOC) Vol.4, No.2/3, September 2012

The ACO based MTA has been implemented on the MANET to determine the variation of throughput with the increase of packet sizes between 1-6 Kb. Figure 1 shows the Throughput variation for different packet sizes for MTA. In the same figure the throughput variations for the termite algorithm which does not incorporate efficinet pheromone decay technique is also shown for comparison.

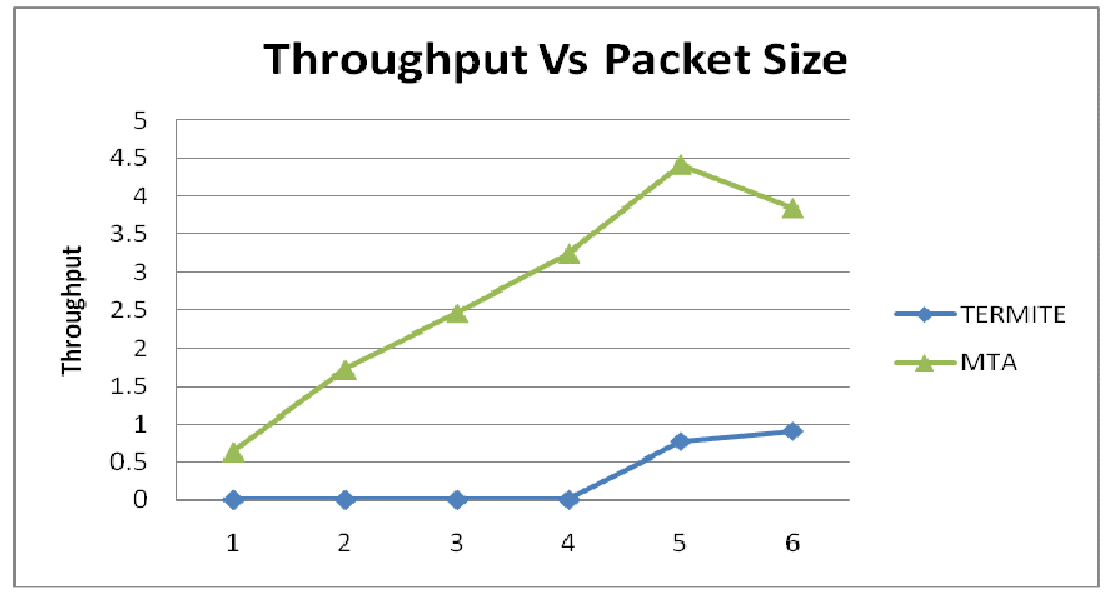

Fig 1 Throughput (Mbps/sec) Vs Packet size (Kb) Analysis

Figure 1 clearly demonstrates that the MTA based MANET shows continuous increase in throughput $\sim 4.0$ times with the packet size, where as the throughput for termite algorithm remains low $<1.0$ and does not show any significant variations with the packet size. This suggests that the MTA algorithm implemented on a MANET with 30 nodes indicates large improvement in the throughput $\sim 75 \%$ with the increase of packet size as compared to termite algorithms

The MTA implemented on MANET has been used to determine the end-to-end delay with the increase of packet sizes in the range of 1-6 Kb. Figure 2 shows the end to end delay variations for different packet sizes. In the same figure the end to end delay variations for the termite algorithm which does not incorporate efficinet pheromone decay technique is also shown for comparison.

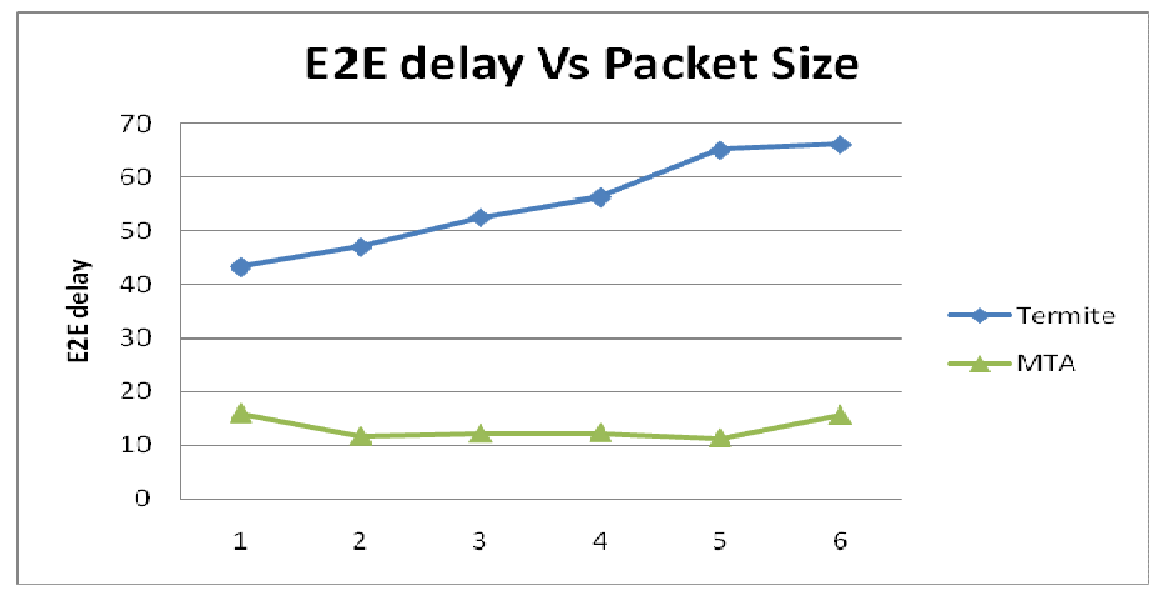

Fig 2 End to End Delay (msec) Vs Packet size (Kb) Analysis 
International journal on applications of graph theory in wireless ad hoc networks and sensor networks (GRAPH-HOC) Vol.4, No.2/3, September 2012

It is seen form the figure 2 that the end to end delay for termite algorithm is $\sim 1.53$ times increase in time delay with the packet size. Where as the end to end delay variation for MTA algorithm < 0.3 and remains almost constant and do not show any significant variations with the packet size This suggests that the MTA algorithm implemented on a MANET with 30 nodes shows reduction in the end-to-end delay $\sim 23 \%$ with the packet size as compared to termite algorithms.

The MTA implemented on MANET has been used to determine the routing over head changes with the increase of packet sizes between 1-6 Kb. Routing overhead is the ratio of number of control packets required for delivery of data .Figure 3 shows the routing over head changes obtained using MTA algorithm for different packet sizes. In the same figure the routing over head changes for the termite algorithm is shown for comparison.

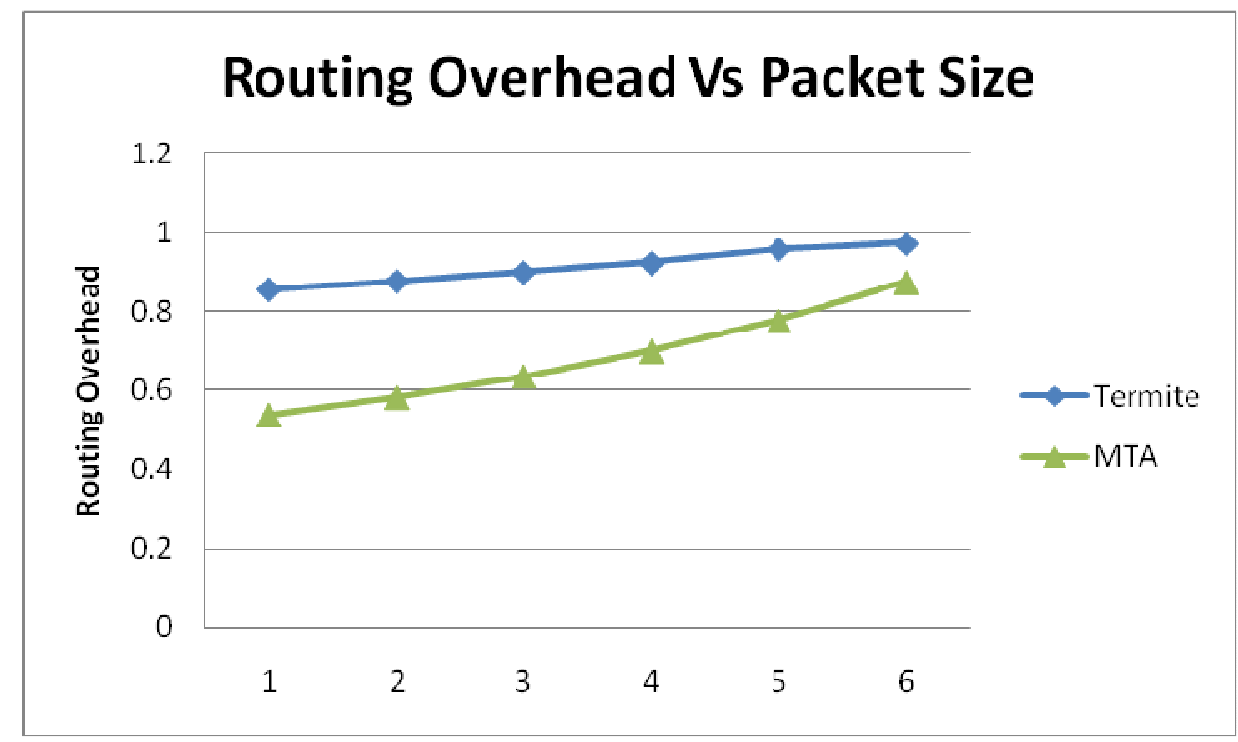

Fig 3 Routing Overhead (packets) Vs Packet size (Kb) Analysis

The figure 3 clearly demonstrates that for termite algorithms the Routing overheads $\sim 1.15$ times increase with the packet size. Where as the routing overheads increases for MTA algorithm $\sim 1.5$ times with the packet size This suggests that the MTA algorithm implemented on a MANET with 30 nodes shows reduction in the overheads $\sim 13 \%$ with the packet size as compared to termite algorithms suggesting that MTA algorithms shows improvement in routing overheads compared to termite algorithms.

\section{CONCLUSION}

From the analysis carried out, the following conclusions are drawn

1. The stagnation problems can be overcome by fine tuning of the Pheromone concentration based on node stability factor.

2. The MTA implemented on MANET is found to perform better for 40 nodes between as the success rates are found to be maximum in this range.

3. The MTA implemented on MANET with fine tuning of pheromone concentration shows significant increase in the throughput $\sim 75 \%$ with the increase of packet size from $1-6 \mathrm{~Kb}$ compared to termite algorithms 
International journal on applications of graph theory in wireless ad hoc networks and sensor networks (GRAPH-HOC) Vol.4, No.2/3, September 2012

4. The MTA implemented on MANET shows significant decrease in End-to-end time delay $23 \%$ with the increase of packet size from 1-6 Kb compared to termite algorithms

5. The MTA implemented on MANET shows a small decrease $\sim 13 \%$ in the routing overheads with the increase of packet size from 1-6 Kb and indicates improvement in routing overheads compared to termite algorithms

\section{REFERENCES}

[1]. C S R.Murthy and B S Manoj, “Ad Hoc Wireless Networks : Architecture and protocols” prentice Hall PTR, 2004

[2]. Elang Huang, Wenjun Hu Jon, Crowcroft Lan, “ Towards commercial Mobile Ad Hoc Network applications: A Radio Dispacth System”, MobiHOC May, 2005,Illinois, USA.

[3]. March Dorigo," Ant Colony Optimisation and swarm intelligence", $4^{\text {th }}$ International workshop, ANTS, 2004, Brussels, Belgium, Sep 2004( Proceedings in LNCS, Berlin:Springer)

[4]. Ducatelle F, Di Caro G and Gambardella L M, "Principles and applications of Swarm Intelligence for adaptive routing in telecommunications networks", Swarm Intelligence, 2010.

[5]. T Stutzle, H H Hoos,"Max-Min Ant system” Future Generation Computing Syst , PP 889-914,2000.

[6]. R Kumar, M K Tiwari and R Shankar, "Scheduling of flexible manufacturing systems: an ant colony optimization approach”, Proceedings Instn Mech Engrs Vol 217, Part B: J Engineering Manufacture, ,pp 1443-1453,2003.

[7]. Kuan Yew Wong, Phen Chiak See, “A New minimum pheromone threshold strategy(MPTS) for Max-min ant system “, Applied Soft computing, Vol 9, pp 882-888 , 2009

[8]. David C Mathew, “ Improved Lower Limits for Pheromone Trails in ACO”, G Rudolf et al(Eds), LNCS 5199, pp 508-517, Springer Verlag, 2008.

[9]. Laalaoui Y, Drias H, Bouridah A and Ahmed R B, “ Ant Colony system with stagnation avoidance for the scheduling of real time tasks", Computational Intelligence in scheduling, IEEE symposium, pp 1-6,2009.

[10]. E Priya Darshini, “ Implementation of ACO algorithm for EDGE detection and Sorting Salesman problem”,International Journal of Engineering science and Technology, Vol 2, pp 2304-2315, 2010

[11]. Alaa Alijanaby, KU Ruhana Kumahamud, Norita Md Norwawi, "Interacted Multiple Ant Colonies optimization Frame work: an experimental study of the evaluation and the exploration techniques to control the search stagnation", International Journal of Advancements in computing Technology Vol 2, No 1, pp 78-85,March 2010 .

[12]. Raka Jovanovic and Milan Tuba, "An ant colony optimization algorithm with improved pheromone correction strategy for the minimum weight vertex cover problem”, Elsvier, Applied Soft Computing, PP 5360-5366, 2011.

[13]. Priyanka Sharma, Dr K Kotecha, “ Optimization in stagnation avoidance of ACO based routing of Multimedia Traffic over Hybrid MANETs", International Journal of computer science and technology, IJCST, Issue 2, ISSN: 2229-4333(print), 0976-8491(online), 2011

[14]. Zar Ch Su Hlaing, May Aye Lhine, "An Ant Colony Optimization Algorithm for solving Traveling Salesman Problem", International Conference on Information Communication and management (IPCSIT), Vol, 6, pp 54-59, 2011.

[15]. Martin Roth and Stephen Wicker "Termite: Emergent Ad-Hoc Networking " Wireless Intelligent Systems Laboratory School of Electrical and Computer Engineering Cornell University Ithaca, New York 14850 USA

[16]. Sharvani G S, Dr. Ananth G and Dr T M Rangaswamy, "Analysis of different pheromone decay techniques for ACO Based routing in Ad Hoc wireless Networks "( communicated). 